\title{
Nonlinear polarization dynamics of Kerr beam self-cleaning in a GRIN multimode optical fiber
}

\author{
K. Krupa ${ }^{1}$, A. Tonello ${ }^{2}$, M. Fabert ${ }^{2}$, V. Couderc ${ }^{2}$, G. Millot ${ }^{3}$, U. Minoni ${ }^{1}$, D. Modotto ${ }^{1}$, and S. Wabnitz ${ }^{1}$ \\ ${ }^{1}$ Dipartimento di Ingegneria dell'Informazione, Università di Brescia, via Branze 38, 25123 Brescia, Italy \\ ${ }^{2}$ Université de Limoges, XLIM, UMR CNRS 7252, 123 Avenue A. Thomas, 87060 Limoges, France \\ ${ }^{3}$ Université Bourgogne Franche-Comté, ICB UMR CNRS 6303, 9 Avenue A. Savary, 21078 Dijon, France \\ Authore-mail address: katarzyna.krupa@unibs.it
}

\begin{abstract}
We experimentally study the polarization dynamics of Kerr beam self-cleaning in a multimode fiber. We reveal that spatial beam cleanup is accompanied by nonlinear polarization evolution and a significant increase of the degree of polarization. (c) 2018 The Author(s) OCIS codes: (190.4370) Nonlinear optics, fibers; (190.3270) Kerr effect; (190.5940) Self-action effects,
\end{abstract}

\section{Introduction}

Research on multimode optical fibers (MMFs) has attracted a strong renewed attention over the last few years, with the first experimental observations of various nonlinear effects including, for example, multimode solitons, geometric parametric instability, spatiotemporal mode-locking [1-3] or Kerr-induced beam self-cleaning. The latter was demonstrated in graded-index multimode fibers (GRIN MMFs), in active Yb-doped MMFs with quasi stepindex profile, and in a multimode PCF [4-6]. Nonlinear mode mixing has been also found to be at the origin of the strong temporal reshaping and pulse shortening which accompany self-cleaning in GRIN MMFs [7].

In standard optical fibers, the polarization components of each guided mode are coupled, owing to the unavoidable presence of small amounts of random birefringence, which is induced by manufacturing imperfections during fiber drawing process, and by externally applied random stress or bending. Linear polarization control in MMFs has been only recently demonstrated by manipulating the spatial wavefront of the input beam [8]. The objective of this paper is instead to point out a new aspect of nonlinear multimode beam propagation, by unveiling the polarization dynamics of the spatial beam self-cleaning induced in MMFs by the Kerr effect.

\section{Experimental results}

We used a standard 12-m long 52/125 graded-index multimode optical fiber (GRIN MMF), that we pumped by a $\mathrm{Nd}$ :YAG microchip laser delivering $500 \mathrm{ps}$ pulses at $1064 \mathrm{~nm}$, with the repetition rate of $500 \mathrm{~Hz}$. We focused a linearly polarized Gaussian beam at the input face of the fiber with a FWHMI diameter of $25 \mu \mathrm{m}$, in order to excite a large number of guided modes. To study the polarization dynamics of nonlinear multimode interactions, we measured all four Stokes parameters by using a polarizer and a quarter-wave plate at the fiber output. We also used an optical spectrum analyzer and a camera to analyze the spectral and spatial properties of the multimode beam.

We placed first a simple polarizer at the output of the fiber. Figure 1 illustrates the evolution of the output power at $1064 \mathrm{~nm}$ normalized to its maximum value, upon a complete rotation of the polarizer axis at low (Fig.1(a)) and high (Fig.1(b)) input power, respectively. Insets of panels (a) and (b) show the corresponding near-field images of the output beam for some selected output polarizer orientations. Whereas, panels (a') and (b') of Fig.1. display the near-field output patterns for the same beam when the polarizer was removed. Panels (a") and (b") of Fig.1 report instead the degree of total polarization (DOP), the degree of linear polarization (DOLP) and the degree of circular polarization (DOCP), for both low and high input power, respectively. These values have been calculated from the measured Stokes parameters after spatial averaging. The light at the output of the fiber appears rather depolarized at low powers and it is characterized by a highly speckled spatial pattern. At $3 \mathrm{~kW}$ peak power, the output beam becomes partially polarized, with up to a four-fold increase of its average degree of polarization, and a dominant linear polarization state. Note that at this power level the expected bell-shaped spatial profile induced by the Kerr beam self-cleaning is only observed around the main polarization axis of the light, while a speckled beam is instead observed on the orthogonal axis.

Interestingly, we noticed that when rotating the linear polarization state of the input laser beam, the direction of the polarization state of the output beam follows these changes, and it also rotates by nearly the same angle, despite the fact that our multimode fiber is intrinsically randomly birefringent. 


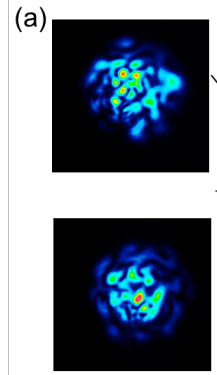

(a')

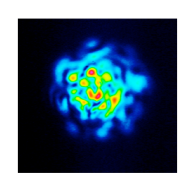

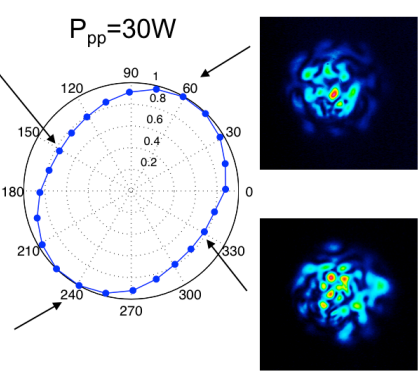

(a")

DOLP $=0.14$

$\mathrm{DOCP}=0.07$

(b)

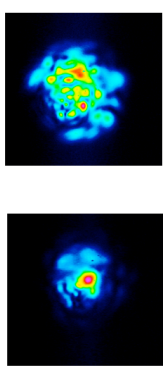

(b')

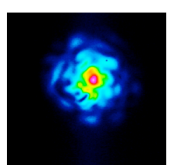

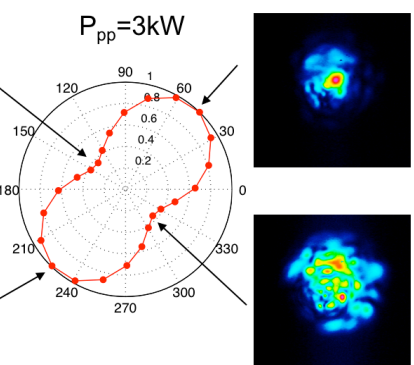

(b")

$$
\begin{aligned}
& \text { DOP }=0.58 \\
& \text { DOLP }=0.57 \\
& \text { DOCP }=0.07
\end{aligned}
$$

Fig.1 Polarization dynamics of beam propagation in GRIN MMF. Normalized power for one polarizer revolution for input peak power (a) $\mathrm{P}_{\mathrm{PP}}=30 \mathrm{~W}$ (linear regime) and (b) $\mathrm{P}_{\mathrm{PP}}=3 \mathrm{~kW}$ (nonlinear regime). Insets: corresponding selected spatial patterns measured at the output face of the fiber. Spatial beam pattern measured without polarizer for (a') $\mathrm{P}_{\mathrm{PP}}=30 \mathrm{~W}$ and ( $\left.\mathrm{b}^{\prime}\right) \mathrm{P}_{\mathrm{PP}}=3 \mathrm{~kW}$. Degree of total polarization DOP, degree of linear polarization DOLP and degree of circular polarization DOCP for (a') $\mathrm{P}_{\mathrm{PP}}=30 \mathrm{~W}$ and (b') $\mathrm{P}_{\mathrm{PP}}=3 \mathrm{~kW}$. Fiber length: $12 \mathrm{~m}$.

\section{Conclusions}

We investigated the polarization behavior of beam self-cleaning in nonlinear GRIN MMFs. We experimentally demonstrated that Kerr-induced beam self-cleaning process reshapes both the transverse profile and the polarization state. The almost depolarized output in linear regime turned at high intensity to almost linear polarization state "attractor" with a DOP equal to 0.6 (4-fold increase when compared to low intensity). Interestingly, we observed this nonlinear polarization effect in the case of both linearly and circularly (not shown in this abstract) polarized input laser beams. Moreover, we also observed the evidence of nonlinear polarization rotation in multimode fibers pumped by linearly polarized beams, which can serve to implement an effective ultrafast saturable absorber mechanism for mode-locked lasers based on multimode fiber.

\section{Acknowledgement}

We acknowledge support from: the European Research Council (ERC) under the European Union's Horizon 2020 research and innovation programme, grant No. 740355 and Marie-Sklodowska-Curie grant No. GA-2015-713694 (MULTIPLY); Horiba Medical and Leukos (MSCA MULTIPLY, BECLEAN project); iXcore research foundation; Labex ACTION program (contract ANR-11-LABX-0001-01).

\section{References}

[1] L. G. Wright, D. N. Christodoulides, F. W. Wise, "Controllable spatiotemporal nonlinear effects in multimode fibres," Nat. Photon. 9, 306 (2015).

[2] K. Krupa, A. Tonello, B. M. Shalaby, A. Barthélémy, V. Couderc, A. Bendahmane, G. Millot, S. Wabnitz, "Observation of geometric parametric instability sidebands by the periodic spatial self-imaging of multimode waves,” Physical Review Letter 116, 183901 (2016).

[3] L. G. Wright, D. N. Christodoulides, F. W. Wise, "Spatiotemporal mode-locking in multimode fiber lasers," Science 358, 94 (2017).

[4] K. Krupa, A. Tonello, B. M. Shalaby, A. Barthélémy, G. Millot, S. Wabnitz, and V. Couderc, "Spatial beam self-cleaning in multimode fiber," Nat. Photon. 11, 237-241 (2017).

[5] R. Guenard, K. Krupa, R. Dupiol, M. Fabert, A. Bendahmane, V. Kermene, A. Desfarges-Barthelemot, J. L. Auguste, A. Tonello, A. Barthélémy, G. Millot, S. Wabnitz, V. Couderc, "Kerr self-cleaning of pulsed beam in ytterbium doped multimode fiber," Opt. Express 25, 47834792 (2017)

[6] R. Dupiol, K. Krupa, A. Tonello, M. Fabert, D. Modotto, S. Wabnitz, G. Millot, V. Couderc, "Interplay of Kerr and Raman beam cleaning with a multimode microstructured fiber,' Opt. Lett. 43, 587-590 (2018).

[7] K. Krupa, A. Tonello, V. Couderc, A. Barthélémy, G. Millot, D. Modotto, S. Wabnitz, "Spatiotemporal light beam compression from complex nonlinear mode mixing,' arXiv:1711.11478 (2017)

[8] W. Xiong, Ch.-W. Hsu, Y. Bromberg, J. E. Antonio-Lopez, R. Amezcua Correa, H. Cao, "Complete Polarization Control in Multimode Fiber with Polarization and Mode Coupling,' arXiv:1709.01029v2 (2017) 